\title{
Experimental and Theoretical Study of 2,6- Difluorophenylnitrene, Its Radical Cation, and Their Rearrangement Products in Argon Matrices
}

\author{
Claudio Carra, Rafael Nussbaum, and Thomas Bally ${ }^{*[a]}$
}

2,6-Difluorophenylnitrene was reinvestigated both experimentally, in Ar matrices at $10 \mathrm{~K}$, and computationally, by DFT and CASSCF/ CASPT2 calculations. Almost-pure samples of both neutral rearrangement products (the bicyclic azirine and the cyclic ketenimine) of a phenylnitrene were prepared and characterized for the first time. These samples were then subjected to X-irradiation in the presence of $\mathrm{CH}_{2} \mathrm{Cl}_{2}$ as an electron scavenger, which led to ionization of the neutral intermediates. Thereby, it was shown

DOI: 10.1002/cphc.200600043

\section{Introduction}

The chemistry observed upon the thermal and photochemical decomposition of aryl azides has been a field of active investigation for decades. ${ }^{[1-3]}$ In part, this persistent interest ${ }^{[4]}$ is driven by the desire to unravel the mechanisms of the complex reactions and to characterize the interesting reactive intermediates that occur therein. However, it is also fueled by the involvement of azides and nitrenes in many practical applications, such as photolithography, ${ }^{[5]}$ preparation of electrically conducting polymers, ${ }^{[6]}$ and photoaffinity labeling of biomolecules. $^{[7,8]}$

Phenylazide $\mathbf{1 a}$, which yields phenylnitrene $\mathbf{2 a}$, the prototypical aryl nitrene, has been the target of numerous experiments using static and time-resolved IR, UV, and ESR spectroscopy. By these experiments, and by quantum chemical calculations, it has been established that the singlet state of phenylnitrene ${ }^{1} \mathbf{2} \mathbf{a}$ undergoes (nearly thermoneutral and reversible) sequential formation of the azirine $\mathbf{3 a}$ and the didehydroazepine $\mathbf{4 a}$, in competition with (irreversible) intersystem crossing to the triplet ground state (Scheme 1). ${ }^{[9]}$ To exploit the bountiful chemistry of the very reactive singlet nitrene in practical applications, its thermal rearrangement must be inhibited. It was found that o-substitution, in particular by fluorine atoms which exert a combined steric and electronic effect, significantly extends the lifetime of the singlet nitrene. ${ }^{[10 a]}$ It is for that reason that derivatives of 2,6-difluorophenylazide $\mathbf{1 b}$ have found important applications as photoaffinity labeling agents. ${ }^{[7]}$ In addition, Morawietz and Sander found that the hitherto elusive azirine intermediate $\mathbf{3 b}$ can be stabilized and characterized in Ar matrices, $^{[11]}$ although transformation into the didehydroazepine $\mathbf{4 b}$ could not be effected. that only the phenylnitrene and the cyclic ketenimine yield stable radical cations, whereas the bicyclic azirine decays to both of these compounds on ionization. The cyclic ketenimine yields a novel aromatic azatropylium-type radical cation. The electronic structure of the title compound is discussed in detail, and its relation to those of the iso- $\pi$-electronic benzyl radical and phenylcarbene is traced.

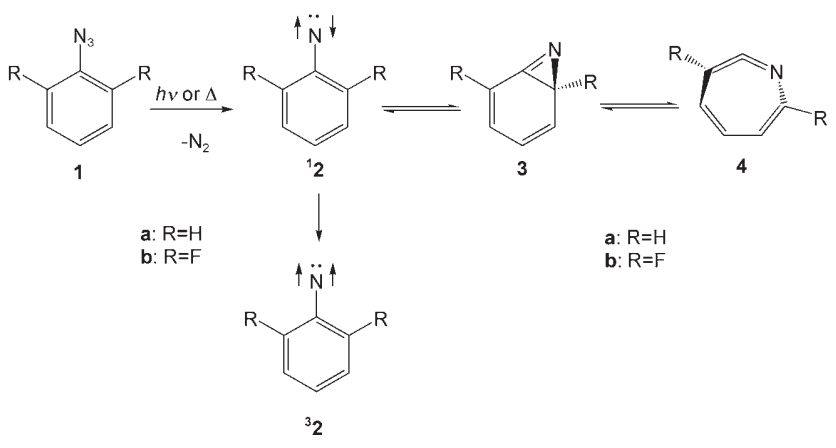

Scheme 1. Decomposition of phenylazide.

Very little is known about the chemistry that azides and nitrenes undergo on reduction or oxidation. The purpose of this work is to fill a part of this gap by investigating the decomposition of phenylazides as well as the fate of the resulting products on ionization in Ar matrices. In particular, we were interested to see how ionization affects the molecular and electronic structure of $\mathbf{2}$ and $\mathbf{4}$, whereby the prospect that the radical cation of $\mathbf{4}$ may be an aromatic species with a closed-shell tropylium-type $\pi$ system appeared most enticing. The difluoro derivatives proved to yield more interesting results, in part be-

[a] Dr. C. Carra, ${ }^{+}$R. Nussbaum, Prof. T. Bally Department of Chemistry, University of Fribourg Ch. du Musée 9, 1700 Fribourg (Switzerland) E-mail: Thomas.Bally@unifr.ch

$\left.{ }^{+}\right]$Current address: USRA, 3600 Bay Area Blvd. Houston, TX 77058-1113

Supporting information for this article is available on the WWW under http://www.chemphyschem.org or from the author. 
cause in this series it is possible to prepare samples of almost pure triplet nitrene $\mathbf{2} \mathbf{b}$, azirine $\mathbf{3} \mathbf{b}$, and, in contrast to earlier experiments, ${ }^{[11]}$ also didehydroazepine $\mathbf{4 b} \mathbf{b}$, which can then be individually subjected to ionization. The experimental results will be complemented by appropriate quantum chemical calculations, which serve to assign the observed spectra and provide an understanding of the molecular and electronic structure of the different reactive intermediates.

\section{Results and Discussion}

\section{Photochemistry of Neutral Compounds}

On short irradiation at $254 \mathrm{~nm}$, matrix-isolated 2,6-difluorophenylazide $\mathbf{1 b}$ undergoes rapid decomposition to yield mainly triplet nitrene ${ }^{3} \mathbf{2} \mathbf{b}_{\text {, }}{ }^{[11]}$ as judged by the UV/Vis and the IR difference spectra $a$ in Figures 1 and 2. The IR spectrum (downward pointing peaks in spectrum a of Figure) is in excellent accord with that predicted by the B3LYP method (blue trace at the bottom), and it appears that ${ }^{3} \mathbf{2} \mathbf{b}$ is the only species that is formed in this step. The UV/Vis spectrum, which is somewhat more detailed and extends further into the UV than that published previously, ${ }^{[11]}$ will be discussed below.

To avoid secondary decomposition we decided to effect the transformation of ${ }^{3} \mathbf{2} \mathbf{b}$ into $\mathbf{3} \mathbf{b}$ by irradiation at the low-energy edge of the first absorption band of ${ }^{3} \mathbf{2} \mathbf{b}$ using an Ar-ion laser. Operating the laser in the single mode did not lead to efficient photolysis, but multimode irradiation $(488-515 \mathrm{~nm}$ ) gave rise to a very clean, albeit still slow, transformation which resulted in the spectra shown as traces $b$ in Figures 1 and 2. Some of the bands in the IR spectrum $b$ are exact mirror images of those in spectrum a, which facilitates their unambiguous assignment to nitrene ${ }^{3} \mathbf{2} \mathbf{b}$. The peaks which rise in the IR spectrum $b$ can equally well be attributed to the azirine $\mathbf{3} \mathbf{b}_{,}^{[11]}$ for example, by comparison with the pattern of bands predicted by B3 LYP/6-31G* (green trace in Figure 2).

After photolysis, the UV spectrum shows a shoulder at $285 \mathrm{~nm}$ and a peak at $235 \mathrm{~nm}$ for $\mathbf{3 b}$, in agreement with earlier observations ${ }^{[11]}$ and with the results of our CASPT2 calculations (Table 1 and green bars in Figure 1).

Upon irradiation at 365 or $254 \mathrm{~nm}, \mathbf{3 b}$ is converted back into nitrene ${ }^{3} \mathbf{2} \mathbf{b}$ rather cleanly. However, we found that on photolysis at $313 \mathrm{~nm}$ a new species forms, next to a small amount of ${ }^{3} \mathbf{2} \mathbf{b}$ (cf. asterisks in the difference spectrum c in Figures 2). This species distinguishes itself by a broad band peaking at about $400 \mathrm{~nm}$ and an IR band at $1830 \mathrm{~cm}^{-1}$, both of which point toward a didehydroazepine structure. ${ }^{[12,13]}$ Indeed, calculations of the IR and UV/Vis spectra of $\mathbf{4 b}$ (purple bars in Figure 1, purple trace in Figure 2) are in excellent agreement with the observed features. Irradiation at $>400 \mathrm{~nm}$ reconverts $\mathbf{4 b}$ to $\mathbf{3 b}$. The IR spectrum of $\mathbf{4 b}$ confirms that the assignment of a peak at $1576 \mathrm{~cm}^{-1}$ in the transient IR spectrum observed in the recent study of Platz et al. ${ }^{[10 b]}$ is correct (the peak appears at $1579 \mathrm{~cm}^{-1}$ in an Ar matrix). However, the similarly intense double peak of $\mathbf{4 b}$ at $1538 / 1527 \mathrm{~cm}^{-1}$ in our matrix spectrum did not show up in the transient IR spectrum, in contrast to

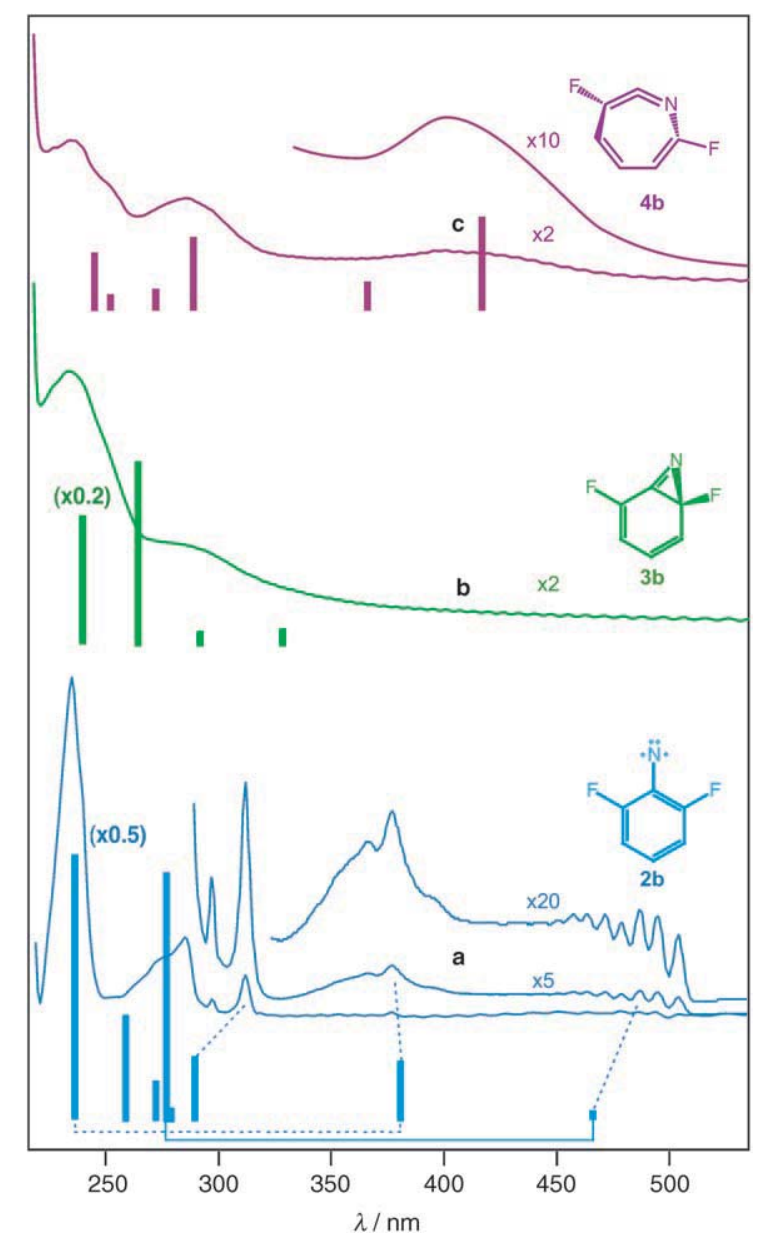

Figure 1. UV/Vis spectra obtained a) after photolysis of 2,6-difluorophenylazide in an Ar matrix at $14 \mathrm{~K}$ and $254 \mathrm{~nm}, \mathrm{~b}$ ) after subsequent irradiation at 485-515 nm (4-W Ar-ion laser in multimode) for $6 \mathrm{~h}$, and c) after irradiation at $313 \mathrm{~nm}$ (medium-pressure $\mathrm{Hg} / \mathrm{Xe} \mathrm{lamp}$, interference filter) for $5 \mathrm{~min}$. The stick spectra represent the electronic transitions for ${ }^{3} \mathbf{2} \mathbf{b}$ (blue), $\mathbf{3 b}$ (green), and $\mathbf{4 b}$ (purple) calculated by the CASSCF/CASPT2 method (see Table 1 and the Supporting Information).

the strong peak at $1411 \mathrm{~cm}^{-1}$ which is clearly seen in Figure 6 of that paper (where it was, however, not assigned to $4 \mathbf{b}$ ). ${ }^{[10 \mathrm{~b}]}$

Thus, we have succeeded in closing the circle and have selectively interconverted all three isomers of the difluoronitrene $\mathbf{2 b}$ while characterizing them by full IR and UV/Vis spectroscopy. Also, the above protocol of irradiations allows us to prepare pure samples of $\mathbf{1} \mathbf{b},{ }^{3} \mathbf{2} \mathbf{b}$, or $\mathbf{3} \mathbf{b}$ and almost pure ones of $\mathbf{4 b}$ (together with a small amount of $\mathbf{1 b}$, which we have never been able to fully exclude; see peaks with asterisks in spectrum c of Figure 2). Scheme 2 sums up our findings on the photochemistry of neutral $\mathbf{1 b}$.

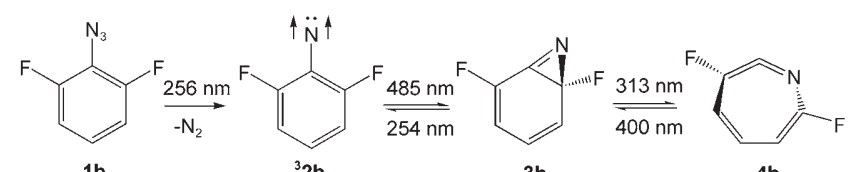

$1 b$

${ }^{3} 2 b$

$3 b$

Scheme 2. Photorearrangement of 2,6-difluorophenylnitrene ${ }^{3} \mathbf{2} \mathbf{b}$. 


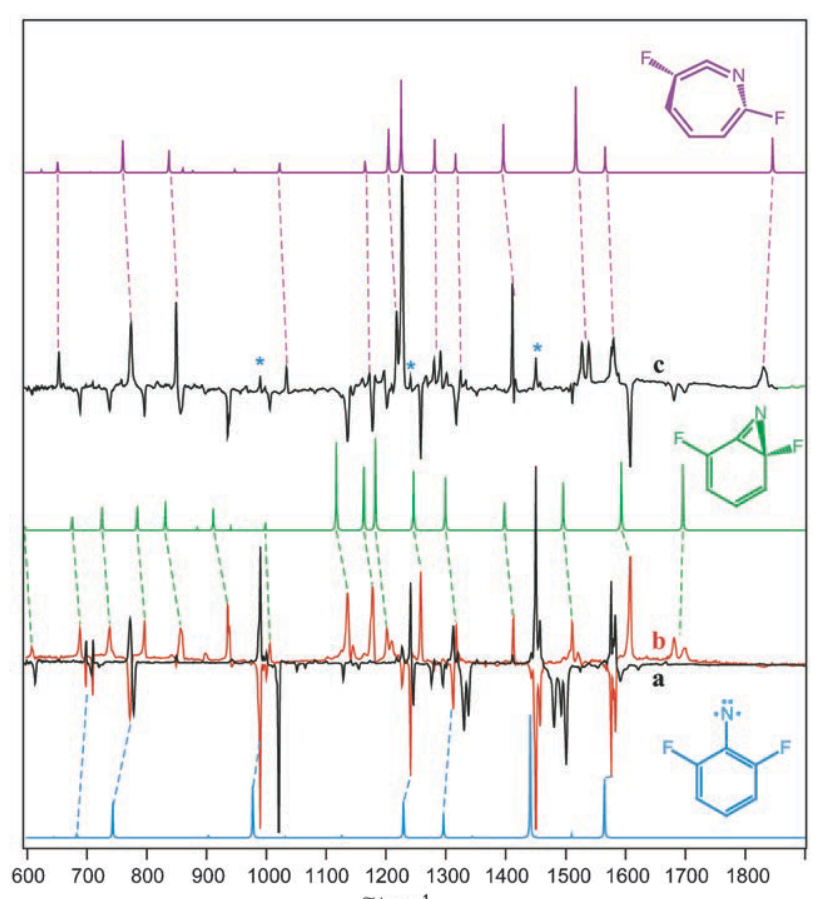

$\tilde{v} / \mathrm{cm}^{-1}$

Figure 2. IR difference spectra for a) photolysis of 2,6-difluorophenylazide in an Ar matrix at $14 \mathrm{~K}$ and $254 \mathrm{~nm}$, b) subsequent irradiation at $485-515 \mathrm{~nm}$ (Ar-ion laser in multimode), and c) irradiation at $313 \mathrm{~nm}$ (medium-pressure $\mathrm{Hg} / \mathrm{Xe}$ lamp, interference filter). The colored traces represent the IR spectra calculated by the B3 LYP/6-31G* method for ${ }^{3} \mathbf{2} \mathbf{b}$ (blue), $\mathbf{3 b}$ (green), and $\mathbf{4 b}$ (purple). The asterisks in spectrum $c$ indicate the major peaks of ${ }^{3} \mathbf{2} \mathbf{b}$, which also forms on the photolysis of $\mathbf{3 b}$ at $313 \mathrm{~nm}$.

\section{The Electronic Structure of Phenylnitrene and Its Isomers}

\section{Triplet 2,6-Difluorophenylnitrene ( $32 b)$}

Phenylnitrene is iso- $\pi$-electronic to the benzyl radical and to triplet phenylcarbene, ${ }^{[14]}$ hence we expect some similarities in the spectra of the three compounds. In addition to excitations within the $\pi$ system, ${ }^{3} \mathbf{2 b}$ has transitions that involve electron promotion to and from the singly occupied in-plane orbital $\left(p_{N}\right)$. If those transitions involve $\pi$ molecular orbitals ( $\pi-M O s$ ), they are polarized perpendicular to the molecular plane and hence carry small oscillator strengths, but, as in the case of phenylcarbene, $\sigma \rightarrow \sigma$ transitions may give rise to additional bands in the spectrum. We have examined the excited-state electronic structure of ${ }^{3} \mathbf{2} \mathbf{b}$ by CASSCF/CASPT2 calculations, the results of which are listed in Table 1 and are represented schematically (blue bars) in Figure 1. The MOs that are involved in the observed transitions are depicted in Figure 3.

Inspection of Table 1 reveals the two pairs of excitations, marked by the arrows in Figure 3, that are typical for the benzyl radical " $\pi$ topology" of ${ }^{3} 2 b$ : the $2 a_{2} \rightarrow 4 b_{2}$ and $4 b_{2} \rightarrow 3 a_{2}$ excitations form negative and positive combinations that give rise to the transitions from the ${ }^{3} A_{2}$ ground state to the first and third ${ }^{3} B_{2}$ excited state, respectively, whereby the transition moments subtract in the first and add up in the second transition. A similar pair of transitions, this time to the second and the fifth ${ }^{3} \mathrm{~A}_{2}$ excited states, arises from combinations of the higher-lying $3 b_{2} \rightarrow 4 b_{2}$ and $4 b_{2} \rightarrow 5 b_{2}$ excitations, except that in this case the high-energy transition contains a significant contribution from excitation to a higher-lying configuration $\left(2 \mathrm{a}_{2} \rightarrow\right.$ $\left.3 a_{2}\right)$.

According to CASPT2 these four transitions give rise to some of the most important bands in the electronic absorption spectrum of ${ }^{3} \mathbf{2 b}$, that is, the two weak bands in the visible region (shown in Figure 1 expanded 20 times), a stronger band at about $290 \mathrm{~nm}$, and the very intense one that is predicted and observed around $235 \mathrm{~nm}$. Further notable excitations within the space of $\pi$-MOs are those leading to the $3^{3} \mathrm{~A}_{2}$ and $4^{3} \mathrm{~A}_{2}$ states, the latter of which may be responsible for the highenergy shoulder on the 290-nm band. (Transitions to the higher-lying ${ }^{3} \mathrm{~B}_{2}$ states are too weak or at too high energy to contribute to the observed spectrum.)

The only remaining unassigned band is the sharp one at $312 \mathrm{~nm}$ with an associated vibronic progression at $296 \mathrm{~nm}$ $\left(+1630 \mathrm{~cm}^{-1}\right)$. The closest transition predicted by CASPT2 involves excitation from the sp lone pair $\left(16 a_{1}\right)$ to the in-plane p-AO $\left(11 b_{1}\right)$ of the $N$ atom $\left(1^{3} A_{2} \rightarrow 2^{3} B_{2}\right)$, but that prediction would be off by $0.3 \mathrm{eV}$ so we sought additional support for it by carrying out separate calculations on that excited state. Although it is not the lowest one of its symmetry, it was possible to converge single-determinant DFT calculations to the $16 \mathrm{a}_{1} \rightarrow$ $11 b_{1}$ excited configuration, because the corresponding wave function (or density) is sufficiently different from that of the lower-lying $\pi \rightarrow \sigma$ excited $1^{3} \mathrm{~B}_{2}$ state. According to the B3LYP method, the resulting state lies $3.92 \mathrm{eV}(315 \mathrm{~nm})$ above the $1^{3} A_{2}$ ground state of ${ }^{3} \mathbf{2} b$ and its equilibrium geometry differs only marginally from that of the ground state, which would explain the occurrence of the intense, sharp 0-0 transition observed at $312 \mathrm{~nm}$.

Several $\pi \rightarrow \sigma$ or $\sigma \rightarrow \pi$ excitations are predicted to occur above $200 \mathrm{~nm}$, but the corresponding transitions are either dipole forbidden $\left({ }^{3} \mathrm{~A}_{2} \rightarrow{ }^{3} \mathrm{~A}_{1}\right)$ or associated with very weak oscillator strengths (transitions to ${ }^{3} \mathrm{~B}_{1}$ states), so they are not expected to contribute to the observed spectrum.

\section{7-Aza-1,5-difluoro-bicyclo[4.1.0]hepta-2,4,6-triene (3b)}

The UV spectrum of azirine $\mathbf{3} \mathbf{b}$ consists of a shoulder around $300 \mathrm{~nm}$ and a slightly asymmetric band peaking at $235 \mathrm{~nm}$. The CASSCF/CASPT2 calculations (see Supporting Information; green bars in Figure 1) predict two weak transitions in the region of the broad shoulder, followed by a medium-intensity transition at $264 \mathrm{~nm}$ and a very strong one at $240 \mathrm{~nm}$. Probably the 264-nm transition is associated with the $235-\mathrm{nm}$ band (prediction too low by $0.58 \mathrm{eV}$ ), while the strong $240-\mathrm{nm}$ transition escapes observation in our experiment, that is, it lies below $220 \mathrm{~nm}$ (error in the prediction $\geq 0.47 \mathrm{eV}$ ). The MOs involved in these transitions are shown in the Supporting Information.

\section{1-Aza-2,6-difluorocyclohepta-2,4,6,7-tetraene (4b)}

The cyclic ketenimine distinguishes itself by a broad band peaking at about $405 \mathrm{~nm}$, followed by bands at 285 and $235 \mathrm{~nm}$ (perhaps with a shoulder at ca. $250 \mathrm{~nm}$ ). The CASSCF/ 


\begin{tabular}{|c|c|c|c|c|c|c|}
\hline State & $\begin{array}{l}\Delta E_{\text {CASSCF }} \\
{[\mathrm{eV}]}\end{array}$ & $\begin{array}{l}\Delta E_{\text {CASPT2 }} \\
{[\mathrm{eV}]}\end{array}$ & $\begin{array}{l}\text { Ref. } \\
\text { weight }^{[b]}\end{array}$ & $\begin{array}{l}\lambda \\
{[\mathrm{nm}]}\end{array}$ & $f^{[c]}$ & $\begin{array}{l}\text { Major } \\
\text { configurations }\end{array}$ \\
\hline $1^{3} \mathrm{~A}_{2}$ & 0.00 & 0.00 & 0.75 & - & - & $87 \%$ ground config. \\
\hline $1^{3} \mathrm{~B}_{2}$ & 3.63 & 2.66 & 0.741 & 466 & $6.8 \times 10^{-4}$ & $\begin{array}{l}64 \% 2 \mathrm{a}_{2} \rightarrow 4 \mathrm{~b}_{2} \\
-16 \% 4 \mathrm{~b}_{2} \rightarrow 3 \mathrm{a}_{2}\end{array}$ \\
\hline $2^{3} \mathrm{~A}_{2}$ & 3.88 & 3.26 & 0.741 & 380 & $1.3 \times 10^{-2}$ & $\begin{array}{l}56 \% 3 \mathrm{~b}_{2} \rightarrow 4 \mathrm{~b}_{2} \\
+27 \% 4 \mathrm{~b}_{2} \rightarrow 5 \mathrm{~b}_{2}\end{array}$ \\
\hline $1^{3} \mathrm{~B}_{1}$ & 4.89 & 4.24 & 0.751 & 293 & $2.2 \times 10^{-4}$ & $\begin{array}{l}67 \% 2 a_{2} \rightarrow 11 b_{1} \\
-14 \%\left[3 b_{2} \rightarrow 11 b_{1}+2 a_{2} \rightarrow 4 b_{2}\right]\end{array}$ \\
\hline $2^{3} \mathrm{~B}_{2}$ & 5.01 & 4.28 & 0.748 & 289 & $1.4 \times 10^{-2}$ & $87 \% 16 a_{1} \rightarrow 11 b_{1}$ \\
\hline $3^{3} \mathrm{~A}_{2}$ & 5.27 & 4.46 & 0.737 & 278 & $3.1 \times 10^{-3}$ & $\begin{array}{l}42 \% 2 a_{2} \rightarrow 3 a_{2} \\
-24 \%\left[4 b_{2} \rightarrow 3 a_{2}+2 a_{2} \rightarrow 4 b_{2}\right]\end{array}$ \\
\hline $1^{3} \mathrm{~A}_{1}$ & 4.45 & 4.48 & 0.748 & 277 & 0.0 & $84 \% 3 b_{2} \rightarrow 11 b_{1}$ \\
\hline $3^{3} \mathrm{~B}_{2}$ & 5.96 & 4.47 & 0.736 & 277 & $5.3 \times 10^{-2}$ & $\begin{array}{l}54 \% 4 \mathrm{~b}_{2} \rightarrow 3 \mathrm{a}_{2} \\
+18 \% 2 \mathrm{a}_{2} \rightarrow 4 \mathrm{~b}_{2}\end{array}$ \\
\hline $2^{3} B_{1}$ & 5.22 & 4.55 & 0.745 & 272 & $8.6 \times 10^{-3}$ & $84 \% 16 a_{1} \rightarrow 4 b_{2}$ \\
\hline $4^{3} \mathrm{~A}_{2}$ & 6.12 & 4.79 & 0.721 & 259 & $2.3 \times 10^{-2}$ & $\begin{array}{l}39 \%\left[4 b_{2} \rightarrow 3 a_{2}+2 a_{2} \rightarrow 4 b_{2}\right] \\
-31 \% 2 b_{2} \rightarrow 4 b_{2}\end{array}$ \\
\hline $4^{3} \mathrm{~B}_{2}$ & 7.05 & 4.47 & 0.736 & 277 & $1.5 \times 10^{-4}$ & $\begin{array}{l}35 \% 2 \mathrm{a}_{2} \rightarrow 5 \mathrm{~b}_{2} \\
35 \% 2 \mathrm{~b}_{2} \rightarrow 3 \mathrm{a}_{2}\end{array}$ \\
\hline $1^{3} \mathrm{~B}_{1}$ & 4.34 & 4.57 & 0.751 & 271 & $2.3 \times 10^{-4}$ & $81 \% 2 a_{2} \rightarrow 11 b_{1}$ \\
\hline $4^{3} \mathrm{~A}_{2}$ & 6.12 & 4.79 & 0.721 & 259 & $2.3 \times 10^{-2}$ & $\begin{array}{l}39 \%\left[4 \mathrm{~b}_{2} \rightarrow 3 \mathrm{a}_{2}+2 \mathrm{a}_{2} \rightarrow 4 \mathrm{~b}_{2}\right] \\
-31 \% 2 \mathrm{~b}_{2} \rightarrow 4 \mathrm{~b}_{2}\end{array}$ \\
\hline $5^{3} \mathrm{~A}_{2}$ & 7.30 & 5.25 & 0.704 & 236 & $1.1 \times 10^{-1}$ & $\begin{array}{l}40 \% 4 b_{2} \rightarrow 5 b_{2} \\
-16 \% 3 b_{2} \rightarrow 4 b_{2} \\
-13 \% 2 a_{2} \rightarrow 3 a_{2}\end{array}$ \\
\hline $4^{3} B_{2}$ & 7.52 & 5.56 & 0.717 & 223 & $7.0 \times 10^{-3}$ & $\begin{array}{l}36 \% 2 \mathrm{a}_{2} \rightarrow 5 \mathrm{~b}_{2} \\
31 \% 2 \mathrm{~b}_{2} \rightarrow 3 \mathrm{a}_{2}\end{array}$ \\
\hline $6^{3} \mathrm{~A}_{2}$ & 7.64 & 5.89 & 0.714 & 211 & $1.6 \times 10^{-2}$ & $\begin{array}{l}28 \% 2 b_{2} \rightarrow 4 b_{2} \\
-14 \%\left[3 b_{2} \rightarrow 4 b_{2}+2 a_{2} \rightarrow 3 a_{2}\right]\end{array}$ \\
\hline
\end{tabular}

[a] Based on a CASSCF(14,12)/ANO-S wave function at the B3LYP/6-311 $+\mathrm{G}(3 \mathrm{~d}, 2 \mathrm{p})$ geometry; to eliminate intruder states, a level shift of $0.2 \mathrm{~h}$ was applied in the CASPT2 calculations. [b] Weight of the zero-order CASSCF in the CASPT2 wave function. [c] Oscillator strength for electronic transition. [d] Electron excitations within the active space of orbitals depicted in Figure 3.

CASPT2 calculations (see Supporting Information; purple bars in Figure 1) indicate that the broad visible band encompasses two transitions that involve mainly the $\mathrm{HOMO}$ and the lowest two virtual MOs, which are centered in the bent allene moiety (see Supporting Information). The next strong transition that coincides with the $285-\mathrm{nm}$ band involves a $\pi \rightarrow \sigma$ transition. The predictions for the higher excited states are too uncertain to allow any definitive assignments. Also, we note that the relative transition moments appear to be predicted poorly by the CASSCF method in this case. However, the excited-state electronic structure is quite sensitive to details of the geometry, and the discrepancies between observed and calculated excitations could also be due to the fact that B3LYP does not yield a very good geometry for this unusual molecule.

\section{Radical Cations}

X-irradiation of a matrix containing azide $\mathbf{1 b}$ led to the spectral changes shown in traces a of Figures 4 and 5 . Clearly the IR bands of the azide "marked ' $A$ ' in Figure 5 " decrease in the process, that is, $\mathbf{1} \mathbf{b}$ is consumed, in addition to the electron scavenger $\mathrm{CH}_{2} \mathrm{Cl}_{2}$ (peaks marked with asterisks), so we conclude that $\mathbf{1 b}$ has indeed been ionized. Both spectra show the appearance of nitrene ${ }^{3} \mathbf{2} \mathbf{b}$ (blue dashed trace in Figure 4; peaks marked " $\mathrm{N}$ " in Figure 5), in addition to a further product

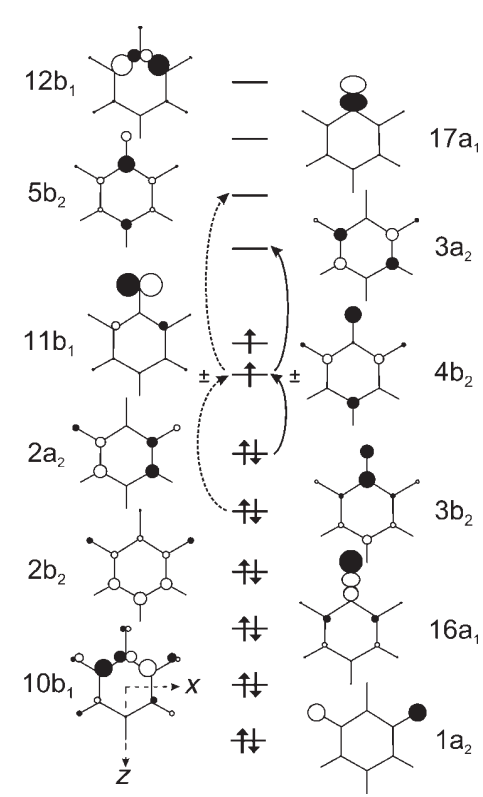

Figure 3. B3 LYP Kohn-Sham MOs of triplet 2,6-difluorophenylnitrene ${ }^{\mathbf{3}} \mathbf{2} \mathbf{b}$. The arrows denote the pairs of electron promotions that lead to excited configurations, which combine to give the leading ${ }^{3} \mathrm{~B}_{2}$ (solid arrows) and ${ }^{3} \mathrm{~A}_{2}$ excited states (dashed arrows, see Table 1). 


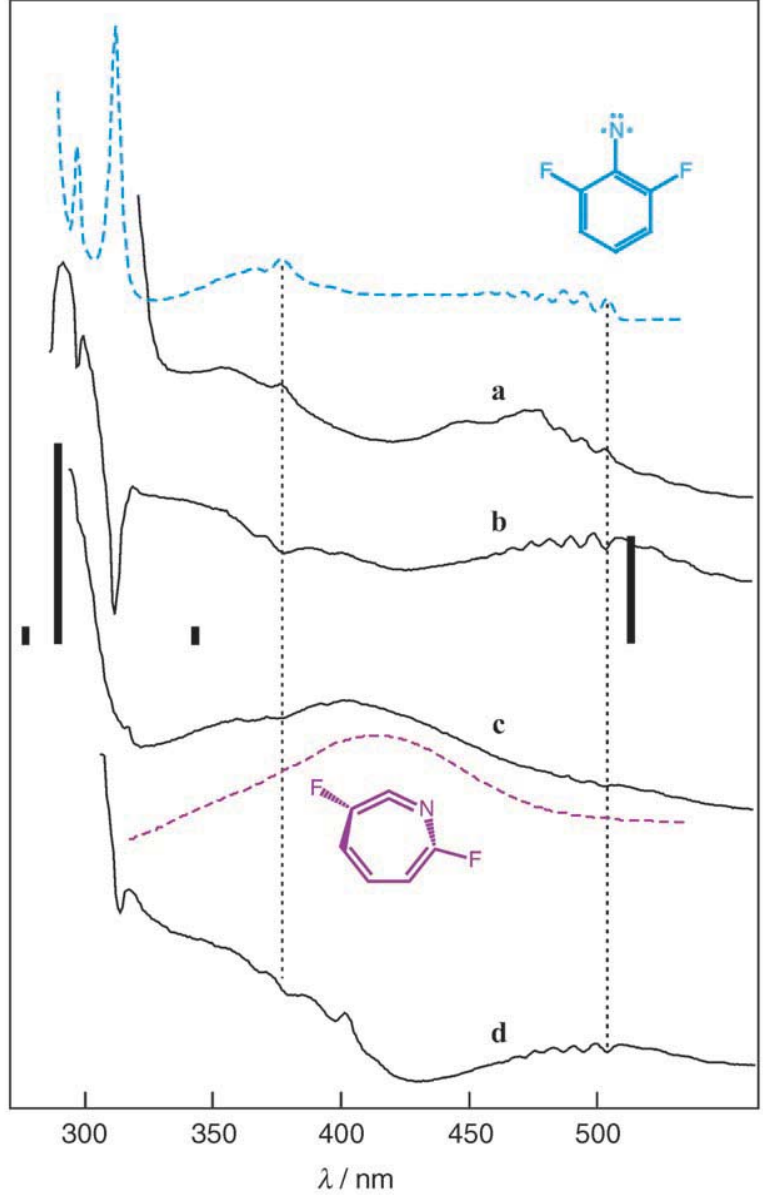

Figure 4. UV/Vis difference spectra for X-irradiation of matrix-isolated azide $\mathbf{1 b}(\mathrm{a}),{ }^{3} \mathbf{2} \mathbf{b}(\mathrm{b}), \mathbf{3} \mathbf{b}(\mathrm{c})$, and $\mathbf{4 b}$ (d). For comparison the spectra of ${ }^{3} \mathbf{2} \mathbf{b}$ (blue) and $\mathbf{4 b}$ (purple) are reproduced with dashed lines.

(or more) with broad absorptions peaking around 350 and $480 \mathrm{~nm}$ and IR bands at 1658, 1430, 1146, 941, and $835 \mathrm{~cm}^{-1}$.

The same group of new IR peaks and similar UV/Vis bands also arise on X-irradiation of a matrix containing only nitrene ${ }^{3} \mathbf{2 b}$ (difference spectra $b$ in Figures 4 and 5), whereby the bands of ${ }^{3} \mathbf{2} \mathbf{b}$, which had increased during X-irradiation of matrix-isolated $\mathbf{1 b}$, decrease (see dashed vertical lines in Figure 4). The pattern of the new IR bands is in good accord with that predicted for the nitrene radical cation $\mathbf{2}^{\mathbf{b}^{+}}$(top red trace and red dashed lines in Figure 5). Therefore, we conclude that on ionization in $\mathrm{Ar}$, azide $\mathbf{1 b}$ spontaneously loses $\mathrm{N}_{2}$ and forms the nitrene radical cation which is reneutralized by the electrons that are continually liberated while the Ar matrix is being irradiated with $\mathrm{X}$-rays, and that do not get scavenged by $\mathrm{CH}_{2} \mathrm{Cl}_{2}{ }^{[15]}$ The two signpost signals of $\mathbf{2} \mathbf{b}^{\cdot+}$ are the strong IR peaks at 1658 and $1449 \mathrm{~cm}^{-1}$.

Next we subjected a sample containing only azirine $\mathbf{3 b}$ to $\mathbf{X}$ irradiation, an operation that resulted in the difference spectra $\mathrm{c}$ in Figures 4 and 5 . Clearly, the peaks of neutral $3 \mathbf{b}$ (marked " $Z$ " in Figure 5) decrease, while the IR peaks assigned to the nitrene radical cation $\mathbf{2}^{\mathbf{b}^{+}}$rise. However, this time no neutral nitrene is formed. Instead we find the bands of the ketenimine $\mathbf{4 b}$ (marked " $\mathrm{K}$ " in Figure 5), while the typical broad

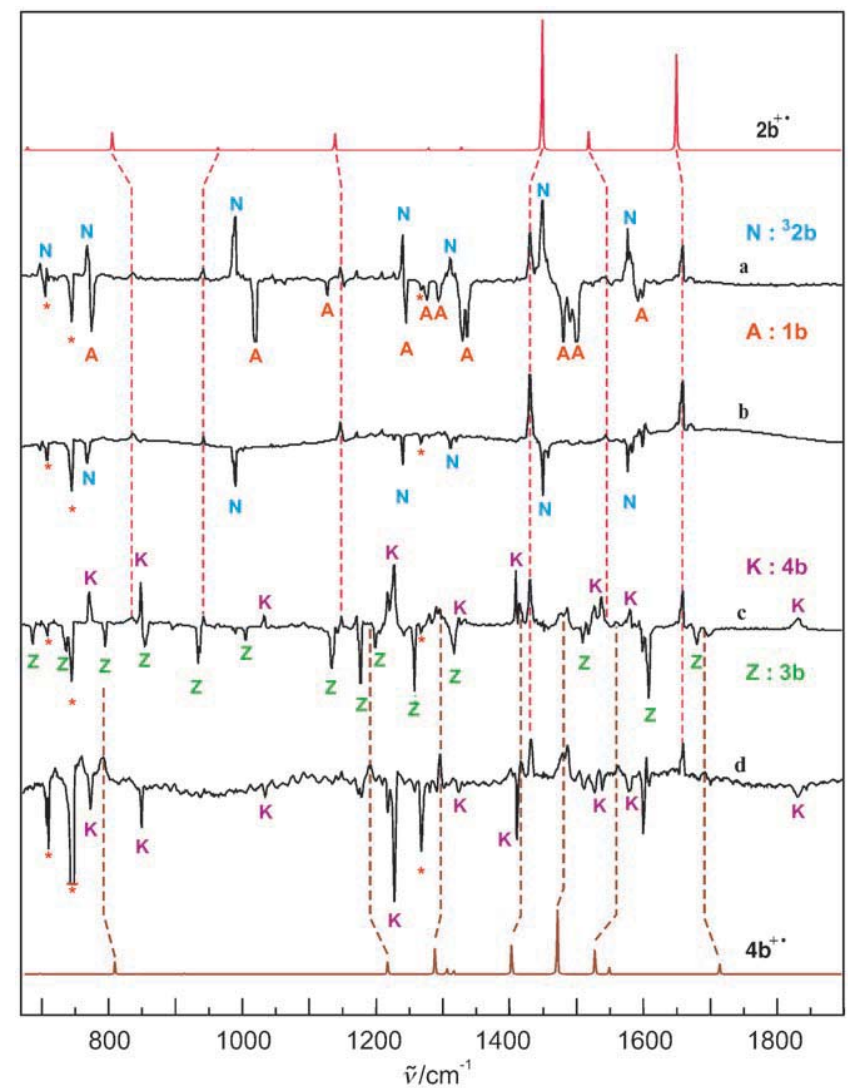

Figure 5. IR difference spectra for X-irradiation of matrix-isolated azide $\mathbf{1 b}$ (a), ${ }^{3} \mathbf{2 b}(\mathrm{b}), \mathbf{3 b}(\mathrm{c})$, and $\mathbf{4 b}(\mathrm{d})$. Blue letters $\mathrm{N}$ denote peaks belonging to $\mathrm{ni}-$ trene ${ }^{3} \mathbf{2} b$, orange $A$ to azide $\mathbf{1 b}$, green $Z$ to azirine $\mathbf{3} b$, and purple $K$ to ketenimine $\mathbf{4} \mathbf{b}$. The red trace at the top and the brown trace at the bottom represent the spectra of $\mathbf{2} \mathbf{b}^{\cdot+}$ and $\mathbf{4 b}^{\mathbf{*}}$, calculated by B3LYP/6-31G*. Dashed lines of the same colors connect these to individual peaks in the experimental spectra.

absorption spectrum of $\mathbf{4 b}$ (dashed purple line in Figure 4) clearly manifests itself in the UV/Vis spectrum. Thus, on ionization, azirine $\mathbf{3 b}$ decays simultaneously by opening the $\mathrm{C}-\mathrm{N}$ bond to give the nitrene radical cation and by opening the bridging $\mathrm{C}-\mathrm{C}$ bond to yield the ketenimine $\mathbf{4 b}$.

Of course, if $\mathbf{4 b}$ is present after ionization, its radical cation $\mathbf{4 b}^{\mathbf{}^{+}}$should also be found. Indeed, the difference spectrum c in Figure 5 shows some upward-pointing peaks that we cannot attribute to any of the products hitherto observed (in particular a double peak around $1480 \mathrm{~cm}^{-1}$ ), but this evidence is not sufficient to provide a basis for an unambiguous assignment. Thus, we finally turned to irradiating a matrix containing predominantly the didehydroazepine $\mathbf{4 b}$, which resulted in the difference spectra $d$ in Figures 4 and 5. The bands of $\mathbf{4 b}$ (marked "K" in Figure 5) decrease, so ionization was successful. Among the new peaks formed in this experiment, those of the nitrene radical cation $\mathbf{2}^{\mathbf{*}}{ }^{+}$(red dashed lines) were again the most prominent, but next to those we found some of the unassigned peaks from the experiment with azirine $\mathbf{3 b}$ (brown dashed lines). At the bottom of Figure 5 we show the calculated spectrum of the radical cation of $\mathbf{4 b}$, the most intense peaks of which can be brought into accord with the major new bands in difference spectrum $d$ that are not due to $\mathbf{2 b}^{\mathbf{}+}$. 
The quantitative agreement admittedly leaves much to be desired, but we can see no other possibility than to assign the new peaks observed on ionization of $\mathbf{3 b}$ and $\mathbf{4 b}$ to $\mathbf{4} \mathbf{b}^{\mathbf{}^{+}}$.

The UV/Vis difference spectra obtained in the latter two experiments do not show any prominent new features, but, as will be shown below, this is not to be expected on the basis of the electronic structure of $\mathbf{4} \mathbf{b}^{\mathbf{}}$. Thus, we conclude that on ionization of $\mathbf{4 b}$ we obtain, in feeble yield, its radical cation. The nitrene radical cation $\mathbf{2} \mathbf{b}^{\mathbf{}}+$ could either be formed by rearrangement of the incipient $\mathbf{4} \mathbf{b}^{\cdot+}$, or directly from residual ${ }^{3} \mathbf{2} \mathbf{b}$ which is always present in samples of $\mathbf{4}$, as is indicated by the decreasing peaks of ${ }^{3} \mathbf{2} b$ in the difference spectrum $d$ in Figure 4.

Scheme 3 sums up the experimental observations reported in this section, which turned up no evidence for the radical cations of the azide $\mathbf{1} \mathbf{b}$ or the azirine $\mathbf{3} \mathbf{b}$, but gave very clear evidence for the nitrene radical cation $\mathbf{2}^{\mathbf{b}^{+}}$and, somewhat less clear, for the targeted aromatic radical cation $\mathbf{4} \mathbf{b}^{\mathbf{}}$.

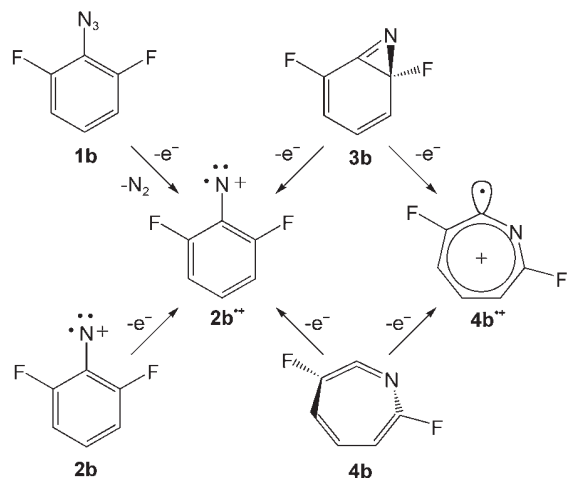

Scheme 3. Radical cations formed on ionization of 2,6-difluorophenylnitrene and its rearrangement products.

\section{Quantum Chemical Calculations on Radical Cations}

B3 LYP/6-31G* calculations of the potential surfaces that join

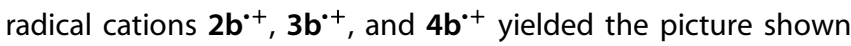
in Figure 6 . In $2 \mathbf{b}^{+}$, about $88 \%$ of the unpaired spin population is on the $\mathrm{N}$ atom, while a similar percentage of the net positive charge is located in the $\mathrm{CF}-(\mathrm{CH})_{3}-\mathrm{CF}$ part of the ring. This indicates a pentadienyl cation bridged by an $\mathrm{R}_{2} \mathrm{C}=\mathrm{N}^{\cdot}$ radical, and the bond-length pattern confirms the dominance of this resonance structure in $\mathbf{2 b}^{\mathbf{b}^{+}}$. (As ionization of $\mathbf{2 b}$ occurs from its essentially nonbonding $\pi$ singly occupied molecular orbital, $4 b_{2}$ in Figure 3 , the geometry changes on ionization are not very pronounced.)

Table 2 lists the electronic transitions calculated for $\mathbf{2}^{\mathbf{}}{ }^{+}$by the CASSCF/CASPT2 method (the same transitions are schematically represented as bars in Figure 4). As in neutral ${ }^{3} \mathbf{2 b}$, transitions that involve electron promotions between $\pi$ - and $\sigma$-MOs are either dipole forbidden $\left(1^{2} B_{1} \rightarrow{ }^{2} B_{2}\right)$ or have very small oscillator strengths $\left(1^{2} B_{1} \rightarrow 1,2^{2} A_{2}\right)$. Thus, the only $\pi \rightarrow \pi$ $\left(1^{2} B_{1} \rightarrow{ }^{2} A_{1} ; 1^{2} B_{1} \rightarrow n^{2} B_{1}\right)$ and $\sigma \rightarrow \sigma$ transitions $\left({ }^{2} B_{1} \rightarrow 2,3^{2} A_{2}\right)$ may contribute to the observed spectrum. These predictions are in qualitative accord with the observation of a broad band with

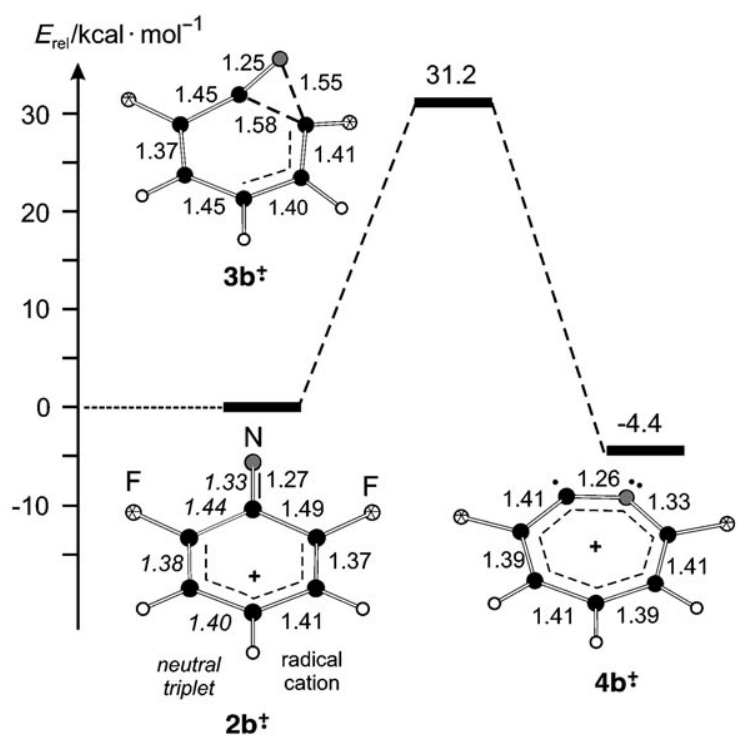

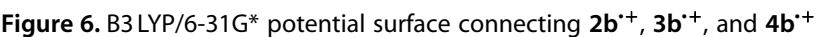
Bond lengths are indicated in $\AA$.

$\lambda_{\max } \approx 500 \mathrm{~nm}$ (probably due to the $1^{2} \mathrm{~B}_{1} \rightarrow 1^{2} \mathrm{~A}_{1}$ transition), a shoulder at about $350 \mathrm{~nm}$ (probably $1^{2} \mathrm{~B}_{1} \rightarrow 2^{2} \mathrm{~B}_{1}$ ), and a stronger band that peaks below $300 \mathrm{~nm}$ (transitions to the $3^{2} \mathrm{~A}_{1}$ and $3^{2} B_{1}$ states). Our spectra turned up no evidence for the weak band predicted at $666 \mathrm{~nm}$, the intensity of which is perhaps overestimated by CASSCF. Just as $\mathbf{2} \mathbf{b}$ is iso- $\pi$-electronic to the benzyl radical, $\mathbf{2}^{\mathbf{b}^{+}}$is iso- $\boldsymbol{\tau}$-electronic to the benzyl cation, the excited-state electronic structure of which has, however, never been assessed conclusively. ${ }^{[16]}$

In contrast to the neutral azirine $\mathbf{3} \mathbf{b}$, no potential energy minimum could be located for its radical cation $3 \mathbf{b}^{\cdot+}$, in spite of the fact that the HOMO of $\mathbf{3 b}$ is largely located in the cyclohexadiene moiety of this compound. Instead, a saddle point was located which IRC calculations proved to connect $\mathbf{2} \mathbf{b}^{\boldsymbol{}^{+}}$to $\mathbf{4 b}^{\mathbf{+}}$. However, the energy of this transition state is so high that an interconversion of these two species is excluded. These findings are in accord with the observation that ionization of a sample of $\mathbf{3 b}$ led exclusively to IR bands associated with $\mathbf{2}^{\mathbf{b}^{\mathbf{}}}$ and $\mathbf{4} \mathbf{b}^{-+}$(plus $\mathbf{4 b}$ which arises by reneutralization), and that ionization of $\mathbf{2} \mathbf{b}$ yielded no $\mathbf{4} \mathbf{b}^{\mathbf{}+}$ and $\mathbf{4} \mathbf{b}$. They also lead us to conclude that the $\mathbf{2} \mathbf{b}^{\mathbf{}}+$ which arises on ionization of $\mathbf{4 b}$ must originate from residual $\mathbf{2} \mathbf{b}$ in that sample.

The most intriguing of the three cations (unfortunately also that for which experimental data are most scarce) is obtained on ionization of $\mathbf{4 b}$. Intriguing, because the very twisted seven-membered ring of the neutral ketenimine becomes planar on ionization and the strong $\mathrm{C}-\mathrm{C}$ bond-length alternation that prevails in $\mathbf{4} \mathbf{b}$ disappears completely: clearly ionization of $\mathbf{4 b}$ has resulted in an aromatic species that is iso- $\pi$ electronic to the tropylium cation. Examination of the electronic wave function of $\mathbf{4 b}^{\mathbf{+}}$ (see Figure 7 ) confirms this diagnosis: the odd electron is located essentially in an in-plane sp hybrid $\mathrm{AO}$ on the hypovalent $\mathrm{C}$ atom next to the $\mathrm{N}$ atom, which itself carries a lone pair. These two AOs form bonding and antibonding linear combinations (MOs 26a' and 27a') and, because the 


\begin{tabular}{|c|c|c|c|c|c|c|}
\hline State & $\begin{array}{l}\Delta E_{\text {CASSCF }} \\
{[\mathrm{eV}]}\end{array}$ & $\begin{array}{l}\Delta E_{\text {CASPT2 }} \\
{[\mathrm{eV}]}\end{array}$ & $\begin{array}{l}\text { Ref. } \\
\text { weight }^{[b]}\end{array}$ & $\begin{array}{l}\lambda \\
{[\mathrm{nm}]}\end{array}$ & $f^{[c]}$ & $\begin{array}{l}\text { Major } \\
\text { configurations }^{[\mathrm{d}]}\end{array}$ \\
\hline $1^{2} \mathrm{~B}_{1}$ & 0.00 & 0.00 & 0.77 & - & - & $82 \%$ ground config. \\
\hline $1^{2} A_{1}$ & 2.34 & 1.86 & 0.76 & 666 & $2.3 \times 10^{-3}$ & $\begin{array}{l}46 \% 2 a_{2} \rightarrow 4 b_{2}(\alpha) \\
-39 \% 2 a_{2} \rightarrow 4 b_{2}(\beta)\end{array}$ \\
\hline $1^{2} \mathrm{~B}_{2}$ & 2.59 & 2.04 & 0.75 & 607 & 0.0 & $76 \% 11 b_{1} \rightarrow 4 b_{2}$ \\
\hline $1^{2} A_{2}$ & 3.83 & 3.15 & 0.75 & 393 & $1.9 \times 10^{-4}$ & $\begin{array}{l}47 \% 2 a_{2} \rightarrow 11 b_{1} \\
-15 \%\left[11 b_{1} \rightarrow 4 b_{2}+2 a_{2} \rightarrow 4 b_{2}\right]\end{array}$ \\
\hline $2^{2} B_{1}$ & 3.80 & 3.59 & 0.76 & 346 & $2.5 \times 10^{-3}$ & $\begin{array}{l}63 \% 3 \mathrm{~b}_{2} \rightarrow 4 \mathrm{~b}_{2}(\alpha) \\
12 \% 3 \mathrm{~b}_{2} \rightarrow 4 \mathrm{~b}_{2}(\beta)\end{array}$ \\
\hline $2^{2} A_{2}$ & 4.91 & 3.94 & 0.75 & 315 & $5.8 \times 10^{-7}$ & $\begin{array}{l}68 \%\left[11 b_{1} \rightarrow 4 b_{2}+2 a_{2} \rightarrow 4 b_{2}\right] \\
+11 \% 2 a_{2} \rightarrow 11 b_{1}\end{array}$ \\
\hline $3^{2} \mathrm{~A}_{1}$ & 4.62 & 4.10 & 0.76 & 302 & $7.4 \times 10^{-3}$ & $82 \% 16 a_{1} \rightarrow 11 b 1$ \\
\hline $3^{2} B_{1}$ & 4.90 & 4.31 & 0.75 & 287 & $4.0 \times 10^{-2}$ & $\begin{array}{l}23 \% 3 b_{2} \rightarrow 4 b_{2}(\alpha) \\
8 \% 3 b_{2} \rightarrow 4 b_{2}(\beta) \\
-22 \% 2 \times\left[2 a_{2} \rightarrow 4 b_{2}\right]\end{array}$ \\
\hline $3^{2} \mathrm{~A}_{2}$ & 5.54 & 4.46 & 0.75 & 278 & $4.8 \times 10^{-3}$ & $80 \% 16 a_{1} \rightarrow 4 b_{2}$ \\
\hline $4^{2} B_{1}$ & 5.20 & 4.47 & 0.75 & 278 & $2.6 \times 10^{-6}$ & $\begin{array}{l}30 \% 2 \mathrm{a}_{2} \rightarrow 3 \mathrm{a}_{2} \\
+16 \% 2 \mathrm{~b}_{2} \rightarrow 4 \mathrm{~b}_{2}(\alpha) \\
+10 \% 2 \mathrm{~b}_{2} \rightarrow 4 \mathrm{~b}_{2}(\beta)\end{array}$ \\
\hline $5^{2} B_{1}$ & 6.29 & 5.07 & 0.74 & 245 & $2.3 \times 10^{-1}$ & $\begin{array}{l}35 \% 2 b_{2} \rightarrow 4 b_{2}(\alpha) \\
+15 \% 2 b_{2} \rightarrow 4 b_{2}(\beta)\end{array}$ \\
\hline \multicolumn{7}{|c|}{$\begin{array}{l}\text { [a] Based on a CASSCF }(13,12) / A N O-S \text { wave function at the B3LYP/6-31G* geometry; to eliminate intruder states, a level shift of } 0.2 \mathrm{~h} \text { was applied in the } \\
\text { CASPT2 calculations. [b] Weight of the zero-order CASSCF in the CASPT2 wave function. [c] Oscillator strength for electronic transition. [d] Electron excita- } \\
\text { tions within the active space of orbitals depicted in the Supporting Information (all the } \pi-M O s \text { and most of the } \sigma \text {-MOs are identical to those shown in } \\
\text { Figure 3). }\end{array}$} \\
\hline
\end{tabular}

former is doubly occupied and the latter only by a single electron, this results in a two-center, three-electron bonding interaction which is presumably responsible for the very short $\mathrm{C}-\mathrm{N}$ bond length. Nominally, the hypovalent $\mathrm{C}$ atom contributes two electrons to the $\pi$ system and the $\mathrm{N}$ atom none (instead a positive charge), but in the end the six $\pi$ electrons occupy the MOs $3 a^{\prime \prime}-5 a^{\prime \prime}$ and are thus delocalized over the entire ring, thus forming an aromatic system.

It is interesting to compare $\mathbf{4 b}^{\mathbf{}^{+}}$to the corresponding planar neutral system which has been discussed in detail previously. ${ }^{[17]}$ One would presume that, to leave the aromatic $\pi$ system intact, an electron will be added to the hybrid AO that (formally) carries the unpaired electron. However, that results in a species with two adjacent lone pairs which suffer enough repulsion to favor a situation where two $\sigma$ electrons are transferred from the hypovalent $C$ atom to the $\pi$ system, which then accommodates eight electrons, that is, becomes antiaromatic. ${ }^{[17]}$ In $\mathbf{4 b}^{\cdot+}$, transfer of the unpaired $\sigma$ electron to the $\pi$ system results in a (nonaromatic) azacycloheptatrienyl radical bearing a positive charge on the hypovalent $\mathrm{C}$ atom. No lonepair repulsion is lost in this process (on the contrary, the twocenter three-electron $\sigma$ bond is broken), and so the resulting species lies $18 \mathrm{kcal} \mathrm{mol}^{-1}$ above $\mathbf{4 \mathbf { b } ^ { + + }}$ in $C_{\mathrm{s}}$ symmetry, and it collapses back to the planar aromatic species if out-of-plane distortions are allowed for.

Inspection of the excited-state electronic structure of $\mathbf{4} \mathbf{b}^{\mathbf{}}$ (see Supporting Information) shows that, apart from two extremely weak $\sigma \rightarrow \pi$ transitions, this aromatic system has no

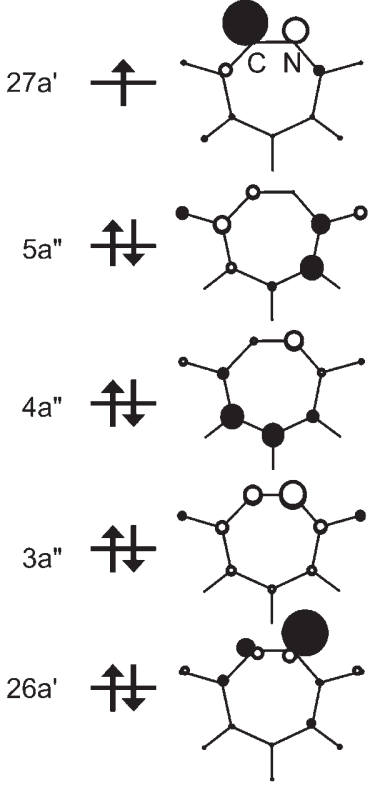

Figure 7. Important occupied valence MOs of $4 \mathbf{b}^{\mathbf{}}$.

bands in the visible part of the spectrum and only one weak $\pi \rightarrow \pi^{*}$ transition above $300 \mathrm{~nm}$. Thus, it is not surprising that no palpable spectral manifestations of $\mathbf{4} \mathbf{b}^{\boldsymbol{}^{+}}$occur in its UV/Vis spectrum (Figure 4), and we must content ourselves with the few IR bands that are attributable to this elusive species (Figure 5) as an experimental proof for its existence. 


\section{Conclusions}

This work demonstrates that it is possible, under favorable circumstances, to generate and characterize both ring-expansion products of singlet phenylnitrenes, that is, the bicyclic azirine and the cyclic ketenimine, by a judicious choice of the wavelengths of irradiation in cryogenic matrices. This has been shown for 2,6-difluorophenylnitrene, where the bicyclic azirine is protected from decay to the cyclic ketenimine by slightly higher barriers. The electronic spectra of the triplet phenylnitrene and its rearrangement products have been recorded down to $220 \mathrm{~nm}$, which permits a more complete discussion of the electronic structure of these species, in particular the phenylnitrene which is iso- $\pi$-electronic to the benzyl radical, than was previously possible.

Almost-pure samples of the three isomers were exposed to $\mathrm{X}$-irradiation in $\mathrm{Ar}$ which leads to the corresponding radical cations. It turns out that on ionization the bicyclic azirine decays spontaneously to the radical cations of the phenylnitrene and the cyclic ketenimine, in accord with calculations which predict that on the radical cation potential surface the bicyclic azirine radical cation is a transition state for the interconversion of the former two species, which were characterized by their IR and UV/Vis spectra.

\section{Experimental and Theoretical Methods}

Azides $\mathbf{1} \mathbf{a}$ and $\mathbf{1 b}$ were placed into a U-tube connected to the inlet system of a closed-cycle cryostat. While this U-tube was held at $-40^{\circ} \mathrm{C}$, a stream of an $\mathrm{Ar} / \mathrm{N}_{2}$ mixture $(85: 15)$ doped with $0.1 \%$ methylene chloride (which serves as the electron scavenger in the ionization step) was passed through it at $\approx 0.1$ Torr, and entrained enough of the azides to give a matrix containing an appropriate amount of the precursor (about $0.1 \mathrm{~mol} \%$ ). Azides were decomposed at $12 \mathrm{~K}$ by short photolysis at $254 \mathrm{~nm}$ (low-pressure $\mathrm{Hg}$ lamp), whereas subsequent photorearrangements were effected by selective photolyses using an Ar laser (multimode: 485-515 nm) or a medium-pressure $\mathrm{Hg} / \mathrm{Xe}$ lamp with a 313-nm interference filter. Electronic absorption spectra were measured on a Perkin-Elmer Lambda 19 instrument, while the IR spectra were measured in an evacuated Bomem DA3 FTIR interferometer.

The geometries of all the stationary points were optimized by the B3 LYP/6-31G* hybrid density functional method ${ }^{[18]}$ using the Gaussian 98 and 03 quantum chemical program packages. ${ }^{[19]}$ Excitation energies and transition moments were computed by the CASSCF/ CASPT2 method $^{[20]}$ as implemented in the MOLCAS program, ${ }^{[21]}$ using atomic natural orbital (ANO)-type basis sets ${ }^{[22]}$ contracted to split valence plus polarization (SVP) quality. The CASSCF wave functions were obtained using the state-averaging technique where all states were equally weighted. In the case of the nitrenes and their radical cations, the active space included the six valence $\pi$ orbitals adding the lone pair of the nitrogen atom and eight electrons.

\section{Acknowledgements}

We are very indebted to Prof. Matthew Platz (Ohio State University) for an initial gift of 2,6-difluorophenylazide with which this study was started. We thank the Swiss National Science Foundation (project no. 200020-105217) for financial support.

Keywords: ab initio calculations - IR spectroscopy $\cdot$ radical ions - reactive intermediates $\cdot$ UV/Vis spectroscopy

[1] W. Lwowski in Nitrenes, Wiley-Interscience, New York, 1970.

[2] E. F. V. Scriven in Azides and Nitrenes: Reactivity and Utility, Academic Press, Orlando, 1984.

[3] M. S. Platz, Acc. Chem. Res. 1995, 28, 487.

[4] C. Carra, T. Bally, A. Albini, J. Am. Chem. Soc. 2005, 127, 5552.

[5] N. J. Clecak, R. J. Cox, W. M. Moreau, Polym. Eng. Sci. 2004, 14, 491.

[6] E. W. Meijer, S. Nijhuis, F. C. B. M. van Vroonhoven, J. Am. Chem. Soc. 1988, 110, 7209.

[7] H. Bayley, Photogenerated Reagents in Biochemistry and Molecular Biology, Elsevier, Amsterdam, 1983.

[8] K. A. Shnapp, E. Poe, E. Leyva, N. Soundararajan, M. S. Platz, Bioconjug. Chem. 1993, 4, 172.

[9] M. S. Platz in Nitrenes (Eds.: R. A. Moss, M. S. Platz, M. Jones), Wiley-Interscience, Hoboken, NJ, 2004, p. 501.

[10] a) N. P. Gritsan, A. D. Gudmundsdóttir, D. Tigelaar, Z. Zhu, W. L. Karney, C. M. Hadad, M. S. Platz, J. Am. Chem. Soc. 2001, 123, 1951; b) S. Mandel, J. Liu, C. M. Hadad, M. S. Platz, J. Phys. Chem. A 2005, 109, 2816.

[11] J. Morawietz, W. Sander, J. Org. Chem. 1996, 61, 4351.

[12] R. J. McMahon, C. J. Abelt, O. L. Chapman, J. W. Johnson, C. L. Kreil, J. P. LeRoux, A. M. Mooring, P. R. West, J. Am. Chem. Soc. 1987, 109, 2456.

[13] J. C. Hayes, R. S. Sheridan, J. Am. Chem. Soc. 1990, 112, 5879.

[14] S. Matzinger, T. Bally, J. Phys. Chem. A 2000, 104, 3544.

[15] T. Bally in Matrix Isolation (Eds.: R. A. Moss, M. S. Platz, M. Jones), WileyInterscience, Hoboken, NJ, 2004, p. 820.

[16] N. Fujisaki, P. Comte, T. Gäumann, Ber. Buns. Ges. Phys. Chem. 1994, 98, 1256.

[17] A. Maltsev, T. Bally, M.-L. Tsao, M. S. Platz, A. Kuhn, M. Vosswinkel, C. Wentrup, J. Am. Chem. Soc. 2004, 126, 237.

[18] B. G. Johnson, P. M. W. Gill, J. A. Pople, J. Chem. Phys. 1993, 98, 5612.

[19] Gaussian 03 (Revision C.01), M. J. Frisch, G. W. Trucks, H. B. Schlegel, G. E. Scuseria, M. A. Robb, J. R. Cheeseman, J. A. Montgomery, Jr., T. Vreven, K. N. Kudin, J. C. Burant, J. M. Millam, S. S. Iyengar, J. Tomasi, V. Barone, B. Mennucci, M. Cossi, G. Scalmani, N. Rega, G. A. Petersson, H. Nakatsuji, M. Hada, M. Ehara, K. Toyota, R. Fukuda, J. Hasegawa, M. Ishida, T. Nakajima, Y. Honda, O. Kitao, H. Nakai, M. Klene, X. Li, J. E. Knox, H. P. Hratchian, J. B. Cross, C. Adamo, J. Jaramillo, R. Gomperts, R. E. Stratmann, O. Yazyev, A. J. Austin, R. Cammi, C. Pomelli, J. W. Ochterski, P. Y. Ayala, K. Morokuma, G. A. Voth, P. Salvador, J. J. Dannenberg, V. G. Zakrzewski, S. Dapprich, A. D. Daniels, M. C. Strain, O. Farkas, D. K. Malick, A. D. Rabuck, K. Raghavachari, J. B. Foresman, J. V. Ortiz, Q. Cui, A. G. Baboul, S. Clifford, J. Cioslowski, B. B. Stefanov, G. Liu, A. Liashenko, P. Piskorz, I. Komaromi, R. L. Martin, D. J. Fox, T. Keith, M. A. Al-Laham, C. Y. Peng, A. Nanayakkara, M. Challacombe, P. M. W. Gill, B. Johnson, W. Chen, M. W. Wong, C. Gonzalez, J. A. Pople, Gaussian, Inc., Wallingford, CT, 2004.

[20] K. Andersson, B. O. Roos in CASPT2, Part 1, Vol. 2, World Scientific, Singapore, 1995, p. 55.

[21] K. Andersson, M. R. A. Blomberg, M. P. Fülscher, V. Kellö, R. Lindh, P.-Å. Malmqvist, J. Noga, J. Olson, B. O. Roos, A. Sadlej, P. E. M. Siegbahn, M. Urban, P.-O. Widmark, MOLCAS, University of Lund, Sweden, 1998.

[22] O. Widmark, P.-Å. Mamqvist, B. O. Roos, Theor. Chim. Acta. 1990, 77, 291.

Published online on May 8, 2006 